\title{
Seguridad alimentaria y sanitaria de Enterococcus provenientes de la provincia del Chubut - Argentina
}

food and health security of Enterococcus from Chubut province - Argentina

${ }^{1}$ Marisol Vallejo ${ }^{a}{ }^{2}$ Pablo Ledesma, ${ }^{3}$ Luis F. Aguirre, Romina B. Parada ${ }^{1}$ y Emilio R. Marguet ${ }^{1}$

\section{RESUMEN}

En este trabajo se estudió la frecuencia y distribución de la resistencia a vancomicina, actividad hemolítica y de gelatinasa en cepas del género Enterococcus aisladas del ambiente, animales salvajes y de cría, origen clínico y de alimentos de la provincia del Chubut, Argentina. Entre los 632 aislamientos, 191 cepas exhibieron al menos uno de los factores de virulencia estudiados; 98 mostraron actividad gelatinasa, 28 resultaron hemolíticas y 110 fueron resistentes a la vancomicina. Enterococcus faecium fue la especie más frecuentemente detectada ( 97 aislamientos), seguido de $E$. faecalis (53 aislamientos), E. durans / hirae (17 aislamientos), E. gallinarum (11 aislamientos), E. columbae (10 aislamientos) y por último E. avium (3 aislamientos).

El uso indiscriminado de antimicrobianos con fines terapéuticos y la administración de factores de crecimiento en la ganadería para mejorar la eficiencia del alimento han provocado un aumento del desarrollo de factores de virulencia, principalmente la resistencia a antibióticos. Por lo tanto, hay una necesidad urgente para estudiar poblaciones de enterococos de diferentes orígenes con el propósito de conocer la relación entre los diferentes grupos y las posibles transferencias de factores de virulencia.

Palabras clave: factores de virulencia, enterococos, ambiente.

\section{ABSTRACT}

The frequency and distribution of vancomycin resistance, hemolytic and gelatinase activity of Enterococcus strains isolated from the environment, wild and breeding animals, food and clinical isolates were studied in Chubut province, Argentine. Among the 632 isolates, 191 strains displayed at least one of the virulence factors studied; 98 showed gelatinase activity, 28 were hemolytic and 110 were resistant to vancomycin. Enterococcus faecium was the most prevalent detected specie (97 isolates), followed by E. faecalis (53 isolates), E. durans/hirae (17 isolates), E. gallinarum (11 isolates), E. columbae ( 10 isolates) and E. avium ( 3 isolates).

The indiscriminate use of antimicrobials for therapeutic aims and the administration of growth promoters to livestock to enhance feed efficiency have caused an increase of the development of virulence factors, mainly antibiotics resistance. Hence, there is an urgent need to study populations of enterococci from different origins in order to know the relation between the different groups and the potential transfers of virulence factors.

Keywords. virulence factors, enterococci, environment.

${ }^{1}$ Universidad Nacional de la Patagonia San Juan Bosco (UNPSJB).

Bióloga. 


\section{INTRODUCCIÓN}

Los enterococos son microorganismos ubicuos, habitantes normales del tracto gastrointestinal de humanos y animales. Debido a su alta tolerancia a condiciones adversas ocupan una gran variedad de nichos conformando parte de la flora del suelo, aguas naturales y plantas (Ogier \& Serror, 2008). Su presencia en alimentos puede resultar perjudicial debido a que son responsables del deterioro de carne, cerveza, jugo de fruta (Foulquié Moreno et al., 2006) o potencialmente beneficiosa como en el caso de quesos que requieren de su actividad metabólica para lograr características sensoriales particulares (Giraffa, 2003).

Como la mayoría de las bacterias lácticas (BL), algunas cepas de enterococos son aplicadas en el proceso de fermentación de alimentos con el propósito de mejorar la calidad sensorial y como probióticos en alimentos y suplementos dietarios (Ogier \& Serror, 2008; Giraffa, 2003). En contraste con estas características positivas, los enterococos son reconocidos como importantes patógenos nosocomiales y están dentro de los organismos más prevalentes en infecciones hospitalarias (Semedo, et al. 2003a; Eaton \& Gasson, 2001).

En los últimos años los enterococos han atraído gran atención debido al aumento de su resistencia natural a varios antimicrobianos y a su capacidad de adquirir nuevos mecanismos de resistencia, entre ellos a los glicopéptidos (vancomicina y teicoplanina) (Franz et al., 2011). Hasta la fecha se han descrito nueve fenotipos de resistencia a glicopéptidos en enterococos: vanA, vanB, vanC, vanD, vanE, vanG, VanL, VanM y VanN (Nilsson, 2012). Los fenotipos vanA y vanB son en el mundo los de mayor distribución y se presentan especialmente en E. faecalis y E. faecium, aunque también han sido reconocidos en otras especies.
Los enterococos resistentes a vancomicna (ERV) se aislaron primero en Europa en 1986 y poco después en Estados Unidos (Cetinkaya et al., 2000). El primer aislamiento de ERV fehacientemente documentado en la Argentina fue en 1998 en una cepa de E. faecium vanA (Marín et al., 1998), posteriormente se comunicaron otros aislamientos de ERV de origen clínico (Miranda et al., 2003) e incluso en la actualidad se han reportado cepas vanA en alimentos de origen animal (Delpech et al., 2012).

Además de su reconocida capacidad para adquirir resistencia los enterococos desarrollan, gracias al intercambio de información genética por conjugación, características que aumentan su virulencia (Khan et al., 2010). Dentro de estas características se puede mencionar: la adherencia a tejidos del hospedante, la invasión y formación de abscesos (Rozdzinski et al., 2001), la modulación de la respuesta inflamatoria, la secreción de productos tóxicos (Eaton \& Gasson, 2001) y la síntesis de enzimas hidrolíticas (Kanemitsu et al., 2001).

Se ha propuesto y demostrado en modelos animales experimentales el intercambio de información genética en el intestino, en consecuencia, existiría la posibilidad de que cepas inocuas provenientes de alimentos se transformen en patógenas gracias a la transferencia de plásmidos (Clewell, 2007). Esta posición es considerada exagerada en la industria alimenticia ya que hasta el momento no se han demostrado casos clínicos de enfermedades transmitidas por alimentos que involucren a los enterococos (Ogier \& Serror, 2008). Sin embargo, es necesario conocer el estado actual de cepas aisladas de alimentos y del ambiente para contar en el futuro con la posibilidad de realizar estudios retrospectivos que permitan afirmar o rechazar la hipótesis de una eventual transferencia de factores de virulencia. 
En la región patagónica existe escasa información sobre la estructura poblacional de las cepas de enterococos (Marguet et al., 2008), así como también de su tasa de resistencia a los antibióticos. Tampoco existen estudios sobre la vía de transmisión de estos microorganismos, resultando necesaria una continua vigilancia para conocer si la transmisión ocurre vía cadena alimentaria o se generan en el ambiente. Por lo expuesto, el aislamiento de cepas de enterococos de diversos ambientes y su posterior estudio de factores de virulencia, brindará información sobre ecología, distribución y características aún no descritas de estos microorganismos en la provincia del Chubut (Patagonia-Argentina).

El presente trabajo se propone como objetivo general estudiar cepas de enterococos de origen humano, alimentario y ambiental, con el propósito de conocer su posible papel como reservorio de genes codificadores de patogenicidad y la influencia de las actividades humanas en la dispersión de cepas con potencial patógeno.

\section{MATERIALES Y MÉTODOS}

\section{Área geográfica del muestreo}

La toma de muestras del estudio se llevó a cabo en las zonas comprendidas del Valle Inferior del Río Chubut, Península Valdés y la costa sur de la provincia del Chubut-Argentina, actualmente denominadas Comarcas VIRCH-Valdés y Río Senguer-Golfo San Jorge respectivamente. Estas zonas geográficas comprenden las localidades de Camarones, Comodoro Rivadavia, Dolavon, Gaiman, Puerto Madryn, Puerto Pirámides, Rada Tilly, Rawson y Trelew. Las muestras se recolectaron desde marzo de 2012 hasta abril de 2013.

\section{Toma de muestras}

Todas las muestras proveniente de hisopados fecales de animales terrestres, sedimento y de agua fueron trasladadas en recipientes estériles, conservándose a $4^{\circ} \mathrm{C}$ hasta el momento de su procesamiento. La toma de muestra proveniente de tracto-gastrointestinal de peces de agua dulce y de ambientes marinos se llevó a cabo según las recomendaciones de Hagi et al. (2004), mientras que para los invertebrados marinos y terrestres se llevaron a cabo según Rahimi et al. (2013).

Las muestras de alimentos se obtuvo con sacabocados estériles y se procesaron dentro de las $4 \mathrm{~h}$.

Las cepas de origen clínico fueron cedidas por el Laboratorio de Patologías Prevalentes y Epidemiología, Hospital Zonal de Trelew de la Provincia del Chubut.

Aislamiento de enterococos de ambientes acuáticos, terrestres, alimentos fermentados y de animales

Las muestras se sembraron en caldo púrpura de bromocresol-azida (Merck, Germany) o caldo etilvioleta azida (EVA) (DIFCO, USA), según el origen de cada muestra. Luego de $24 \mathrm{~h}$ de incubación a $37^{\circ} \mathrm{C}$ los cultivos se repicaron a agar bilis esculina (Merck, Germany), suplementado con ácido nalidíxico $(40 \mu \mathrm{g} / \mathrm{ml})$ y nistatina $(20 \mu \mathrm{g} / \mathrm{ml})$. Todas las muestras se incubaron a $37^{\circ} \mathrm{C}$ durante $24-48 \mathrm{~h}$.

\section{Identificación preliminar y conservación de los aislamientos}

Las colonias obtenidas en los aislamientos se seleccionaron en función de la coloración de Gram, la morfología celular, las pruebas de la catalasa y la oxidasa, crecimiento a $45^{\circ} \mathrm{C}$, a pH 9.6; hidrólisis de la esculina en presencia de sales biliares (40\%), actividad de pirrolidonil aminopeptidasa (PYR) y leucinaaminopeptidasa(LAP).

Las colonias pertenecientes al género Enterococcus se identificaron a nivel de especie mediante fermentación de azúcares según el esquema de Manero y Blanch (1999). 
Las cepas seleccionadas mediante la identificación preliminar se conservaron a $-30^{\circ} \mathrm{C}$ en una suspensión de leche descremada $10 \%$ $(\mathrm{m} / \mathrm{v})$ - glicerol 10\% (v/v).

\section{Evaluación de la seguridad de las cepas}

Con el objeto de determinar indicadores relacionados con factores de virulencia y/o rasgos negativos para la salud y la industria alimenticia, las cepas identificadas, según los procedimientos descritos, fueron sometidas a las siguientes pruebas:

\section{a) Actividad de la gelatinasa}

Para la prueba de la gelatinasa se utilizó un test propuesto por Kanemitsu et al. (2001). Para tal fin se suplementó el agar tripticasa soja (Britania, Argentina) con $0,8 \%(\mathrm{~m} / \mathrm{v})$ de gelatina, las placas se incubaron durante $48 \mathrm{~h}$ a $37{ }^{\circ} \mathrm{C}$ y se revelaron con una solución de tricoloroacético al 20\% (v/v). Las zonas claras alrededor de las cepas se consideraron como positivas.

\section{b) Actividad hemolítica}

La producción de hemolisinas de las cepas aisladas se evaluaron en agar cerebro-corazón (BHI) (Britania, Argentina) suplementado con sangre desfibrinada de conejo al $5 \%$ luego de una incubación a $37^{\circ} \mathrm{C}$ durante $48 \mathrm{~h}$. Los resultados se interpretaron como positivos cuando se observó un halo de hemólisis completa alrededor de las colonias ( $\beta$-hemólisis).

\section{c) Selección de cepas potencialmente resistentes a vancomicina}

Para la búsqueda de microorganismos con potencial resistencia se utilizó el agar bilisesculina (Merck, Germany) suplementado con vancomicina $(6 \mu \mathrm{g} / \mathrm{ml})$ según las recomendaciones de Domig et al. (2003). Todas las cepas que exhibieron desarrollo durante este ensayo se enriquecieron en caldo BHI y se repicaron a placas chromID VRE (Biomérieux, France) según las instrucciones del fabricante.

\section{RESULTADOS}

En total, se aislaron 632 cepas pertenecientes al género Enterococcus, las cuales presentaron los rasgos fenotípicos que son utilizados para distinguir dicho género de otros cocos Grampositivos, catalasa negativos y anaerobios facultativos. Las pruebas diferenciales realizadas fueron: capacidad de crecer en presencia de $6,5 \%$ de $\mathrm{NaCl}$, a $\mathrm{pH}$ 9.6; a $45{ }^{\circ} \mathrm{C}$, capacidad de hidrolizar la esculina en presencia de sales biliares al $40 \%$ y presentar actividad PYR y LAP.

Del total de cepas aisladas, 191 se identificaron de manera preliminar a nivel de especie por medio de los patrones de fermentación de azúcares, por los cuales 97 cepas se clasificaron como E. faecium, 53 como E. faecalis, 17 cepas como E. durans/hirae, 11 como E. gallinarum, 10 cepas como $E$. columbae y 3 cepas como $E$. avium, los resultados se muestran en el gráfico 1. La selección de estas cepas se realizó sobre la base de presentar uno o más de los factores de virulencia estudiados. Con posterioridad se deberán realizar las pruebas genotípicas con cebadores específicos para poder discriminar entre especies que están asociadas a grupos y confirmar las restantes.

En este estudio, del total de microorganismos seleccionados ( $\mathrm{n}=191), 110$ cepas $(58 \%)$ fueron capaces de resistir la concentración de vancomicina empleada (6 $\mu \mathrm{g} / \mathrm{ml})$, aunque cuando se utilizaron las placas comerciales de medio cromogénico VRE no todas las cepas se desarrollaron (datos no mostrados). Esto puede deberse a que el medio comercial empleado está diseñado para aislamientos clínicos, específicamente para diferenciar entre $E$. faecalis y E. faecium e impedir el crecimiento de otras especies de enterococos que presentan resistencia intrínseca a la vancomicina (genotipo 
vanC). De acuerdo a las técnicas utilizadas para detectar resistencia, la mayor frecuencia se presentó en aislamientos provenientes de mamíferos $(n=34)$ en los cuales se incluyen las muestras de origen hospitalario, luego le siguen las muestras de alimentos ( $\mathrm{n}=26)$, invertebrados $(n=23)$, aves $(n=17)$ y en menor proporción en los otros nichos estudiados (Tabla 1).

La actividad de gelatinasa se detectó en 89 cepas (47\%), de las cuales más de la mitad provienen de aves $(n=47)$, en segundo lugar encontramos este rasgo en microorganismos provenientes de alimentos fermentados y las restantes de otros orígenes (Tabla 1).

La actividad hemolítica, como los anteriores ensayos, se evaluaron fenotípicamente en todos los aislamientos, detectándose sólo en 28 cepas provenientes de todos los orígenes evaluados (Tabla 1).

Tabla 1. Factores de virulencia presentes en cepas de enterococos seleccionadas

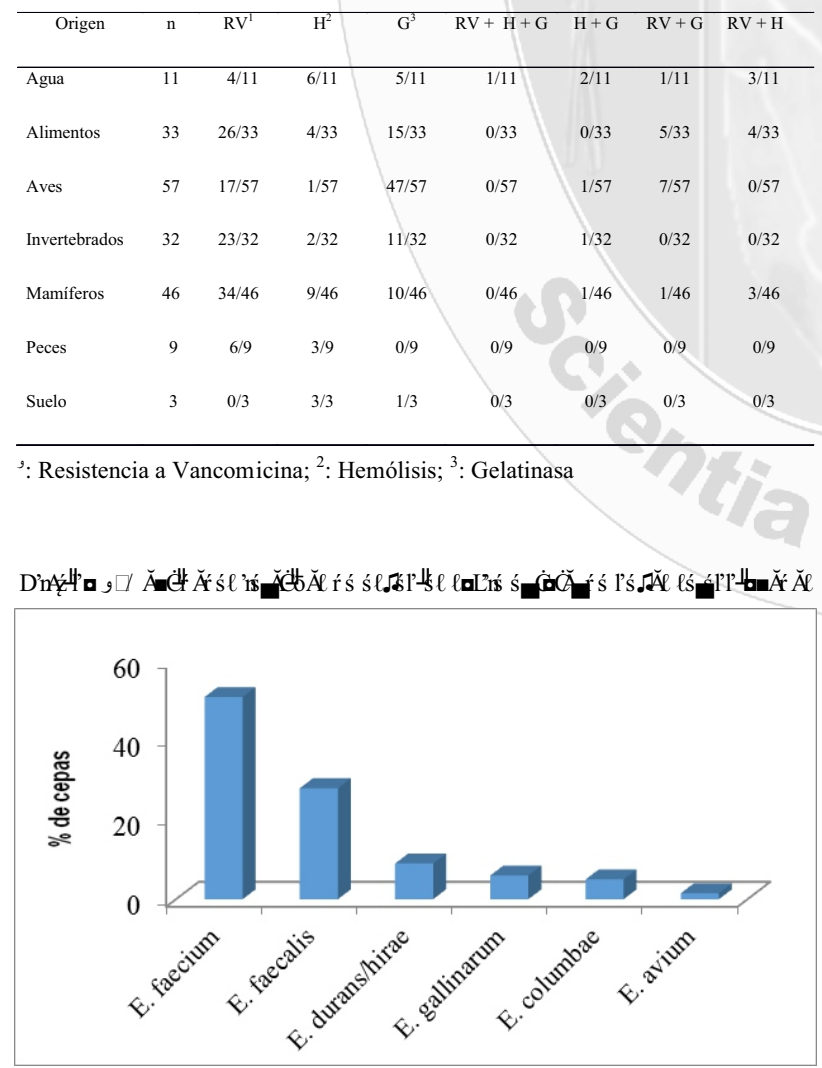

\section{DISCUSIÓN}

Los resultados obtenidos muestran una alta prevalencia de cepas de Enterococcus aisladas de diferentes hábitats, aún en aquellos casos donde las muestras provienen de alimentos elaborados con materia prima pasteurizada, indicando que este género presenta resistencia a los tratamientos térmicos empleados (Giraffa, 2002).

La poca diversidad encontrada a nivel de especie es característica, debido a que E. faecium, $E$. faecalis y E. durans son las más frecuentes en este tipo de nicho ecológico. Estos resultados están de acuerdo con los hallados en diversos trabajos previos, en los cuales $E$. faecalis y $E$. faecium son las especies predominantes en alimentos y animales (Fisher \& Phillips, 2009), mientras que las especies E. hirae, E. gallinarum, E. casseliflavus y otras se han reportado en cuerpos de aguas residuales (Rahimi et al., 2007).

Las BL se consideran microorganismos totalmente inocuos para su incorporación en los alimentos. Sin embargo, en los últimos años se ha cuestionado este estatus GRAS (microorganismos reconocidos como seguros) en el género Enterococcus, ya que algunos autores los consideran microorganismos indeseables, indicadores de contaminación fecal y responsables del deterioro de productos como la carne (Barbosa et al., 2010). Esta controversia se ha visto además acentuada por la desafortunada relevancia clínica que en las últimas décadas han adquirido los enterococos. Estos se han convertido en la tercera causa de enfermedades nosocomiales causando infecciones del tracto urinario, bacteremia y endocarditis (Abriouel et al., 2008). 
Varios son los posibles factores de virulencia que existen, entre los que podemos citar la capacidad hemolítica, la licuefacción de la gelatina y resistencia a la vancomicina. Entre los mencionados, la resistencia a vancomicina es la más preocupante, ya que este antibiótico es una de las últimas opciones terapéuticas en el tratamiento de algunas infecciones por bacterias Gram-positivas (Werner et al., 2008).

La resistencia a la vancomicina y sus análogos se debe a la síntesis de precursores modificados de la pared bacteriana que muestran una baja afinidad por estos glicopéptidos. En el caso de resistencia intrínseca la transmisión es vertical y exhiben un bajo nivel (genotipos vanC) mientras que en los casos extrínsecos, esta propiedad es transmitida en forma horizontal a través de elementos genéticos móviles pudiendo presentar niveles de resistencia alto (genotipos vanA), intermedio (genotipos vanD) o variables (genotipos vanB) (Houben, 2003; Nilsson, 2012).

En la actualidad son abundantes los estudios llevados a cabo en distintas partes del mundo, en los cuales se ha encontrado una alta frecuencia de aislamiento de enterococos resistentes a vancomicina provenientes de muestras clínicas, animales y ambiente entre otros (Delpech et al., 2012; Barbosa, et al., 2010; Kühn et al., 2005). Por otra parte, los estudios epidemiológicos han permitido establecer una fuerte relación entre el uso de antibióticos en la cría de animales y la aparición de cepas resistentes en los alimentos derivados de los mismos, y posteriormente en el tractogastrointestinal de humanos (Van den Bogaard \& Stobberingh, 2000). Sin embargo, hasta la fecha no se ha podido demostrar que las cepas presentes en alimentos puedan ocasionar enfermedades en humanos. Además, estudios recientes indican que las cepas portadoras de caracteres de virulencia forman un grupo genómico diferente de la mayoría de las cepas aisladas de alimentos (Abriouel, et al., 2008).
A partir de estos resultados, se hace indispensable realizar estudios mediante PCR para determinar la presencia de genes relacionados con la resistencia a vancomicina, específicamente los genotipos vanA y vanB de importancia clínica.

Otro factor de virulencia importante de los enterococos constituye la expresión de la gelatinasa, enzima capaz de degradar a la gelatina, caseína, hemoglobina, colágeno y otros péptidos bioactivos. Aunque la actividad de esta enzima es considerada un factor de virulencia en cepas de origen clínico, también se han encontrado en alimentos fermentados (Semedo et al., 2003b; Eaton \& Gasson, 2001; Kanemitsu et al., 2001) y en aves salvajes (Silva et al., 2011). La alta frecuencia encontrada en aves parece ser un rasgo común en este tipo de nicho ecológico, aunque a diferencia de otros estudios, en nuestro caso la actividad gelatinasa no se encuentra restringida a cepas de E. faecalis (datos no mostrados).

La citolisina es una proteína que tiene la capacidad de lisar eritrocitos humanos y además es activa contra otros cocos Gram-positivos, funcionando como un mecanismo de defensa. Se ha demostrado experimentalmente que esta proteína aumenta la patogenicidad en los modelos animales ensayados (Murray, 1990), pero no es un prerrequisito para la virulencia, debido a que cepas de enterococos no hemolíticas pueden también ser causantes de infecciones (Barbosa et al., 2010). Actualmente se sabe que la hemolisina $\beta$ o citolisina es una toxina extracelular sintetizada por numerosos aislamientos de E. faecalis, y que también se reconoce como una bacteriocina (Franz et al., 2003).

En estudios anteriores, Semedo et al. (2003b) examinaron la presencia de diversos factores de virulencia, entre ellos la citolisina, en enterococos aislados de alimentos y muestras 
clínicas. Se observó que la presencia de la citolisina y el potencial hemolítico estaban muy extendidos, con una asociación muy significativa de este factor de virulencia con los aislados clínicos, lo que no ocurría con otros factores de virulencia evaluados (proteasas, adhesinas de superficie, etc), que mostraban una incidencia elevada independientemente de su origen. En nuestro estudio, la incidencia de actividad hemolítica como se mencionó anteriormente se presentó en cepas de diferentes orígenes, no pudiendo establecer una correlación entre este factor de virulencia y el origen de la cepa.

\section{CONCLUSIÓN}

Los enterococos constituyen una fracción importante de la microbiota intestinal autóctona de los mamíferos y otros animales. Una vez liberados al ambiente junto con las excretas, son capaces de colonizar diversos nichos ecológicos gracias a su capacidad para sobrevivir a las condiciones ambientales desfavorables y desarrollarse en ambientes hostiles. A partir del intestino o del medio ambiente, pueden contaminar las materias primas empleadas para la elaboración de los alimentos (especialmente los de origen cárnico y lácteo).

Los resultados obtenidos muestran que los factores de virulencia se encuentran en los enterococos independientemente de su origen o localización (humanos, animales, alimentos y ambiente) sugiriendo que dichos determinantes genéticos son una característica común y ampliamente extendida en este género microbiano.

\section{AGRADECIMIENTOS}

Este trabajo fue financiado con fondos otorgados por la Universidad Nacional de la Patagonia San Juan Bosco al proyecto "Evaluación de la seguridad alimentaria y sanitaria de Enterococcus ambientales" (PI R/7 222/2013).
A la Bioq. Diana Berry, directora del Laboratorio de Patologías Prevalentes y Epidemiología, Hospital Zonal de Trelew de la Provincia del Chubut por las cepas de enterococos.

\section{REFERENCIAS BIBLIOGRÁFICAS}

Abriouel, H., Ben Omar, N., Cobo Molinos, A., Lucas López, R., Grande, M.J., MartínezViedma, P., Ortega, E., Martínez-Cañamero, M. and Gálvez, A. 2008. Comparative analysis of genetic diversity and incidence of virulence factors and antibiotics resistance among enterococcal populations from raw fruit and vegetable foods, water and soil, and clinical samples. Int. J. Food Microbiol. 123:38-49p.

Barbosa, J., Gibbs, P.A. and Teixeira, P. 2010. Virulence factors among enterococci isolated from traditional fermented meat products produced in the North of Portugal. Food Control. 21:651-656p.

Cetinkaya, Y., Falk, P. and Mayhall, C.G. 2000. Vancomycin -resistant enterococci. Clin. Microbiol. Rev. 13:686-707p.

Clewell, D.B. 2007. Properties of Enterococcus faecalis plasmid pAD1, a member of a widely disseminated family of pheromone-responding, conjugative, virulence elements encoding cytolysin. Plasmid. 58:205-207p.

Delpech, G., Pourcel, G., Schell, C., De Luca, M., Basualdo, J., Bernstein, J., Grenovero, S. and Sparo, M. 2012. Antimicrobial resistance profiles of Enterococcus faecalis and Enterococcus faecium isolated from artisanal food of animal origin in Argentina. Foodborne Pathog. Dis. 9:1-12p.

Domig, F.J., Mayer, H.K. and Kneifel, W. 2003. Methods used for the isolation, enumeration, characterization and identification of Enterococcus spp. 1. Media for isolation and enumeration. Int. J. Food Microbiol. 88:147-164p. 
Eaton, T.J. and M.J. Gasson. 2001. Molecular screening of Enterococcus virulence determinants and potential for genetic exchange between food and medical isolates. Appl. Environ. Microbiol. 67:1628-1635p.

Fischer, K. and Phillips, C. 2009. The ecology and virulence of Enterococcus. 2009. Microbiology. 155:1749-1757p.

Foulquié Moreno, M.R., Sarantinopoulos, P., Tsakalidou, E. and De Vuyst, L. 2006. The role and application of enterococci in food and health. Int. J. Food Microbiol. 106:1-24p.

Franz, C.M.A.P., Stiles, M.E., Schleifer, K.H. and Holzapfel, W.H. 2003. Enterococci in foods a conundrum for food safety. Int. J. Food Microbiol. 88:105-122p.

Franz, C.M.A.P., Huch, M., Abriouel, H., Holzapfel, W. and Gálvez, A. 2011. Enterococci as probiotics and their implications in food safety. Int. J. Food Microbiol. 151:125-140p.

Giraffa, G. 2002. Enterococci from foods. FEMS Microbiol. Rev. 744:1-19p.

Giraffa, G. 2003. Functionality of enterococci in dairy products. Int. J. Food Microbiol. 88:215222 p.

Hagi, T., Tanaka, D., Iwamura, Y. and Hoshino, T. 2004. Diversity and seasonal changes in lactic acid bacteria in the intestinal tract of cultured freshwater fish. Aquaculture. 234:335-346p.

Houben, J.H. 2003. The potencial of vancomycin-resistant enterococci to persist in fermented and pasteurized meat products. Int. J. Food Microbiol. 88:11-18p.

Kanemitsu, K., Nischino, T., Kunishima, H., Okamura, N., Takemura, H., Yamamoto, H. et al. 2001. Quantitative determination of gelatinase activity among enterococci. J. Microbiol. Methods. 47:11-6p.
Khan, M.A., Northwood, J.B,. Loor, R.G.J., Tholen, A.T. Riera, E., Falcón, M. et al. 2010. High prevalence of ST-78 infection associated vancomycin-resistant Enterococcus faecium from hospitals in Asunción, Paraguay. Clin. Microbiol. Infect. 16:624-627p.

Kühn, I., Iversen, A., Finn, M., Greko, C., Burman, L.G., Blanch, A.R., et al. 2005. Occurrence and relatedness of vancomycinresistant enterococci in animals, humans, and the environment in different European regions. Appl. Environ. Microbiol.71(9):5383-90p.

Manero, A. and Blanch, A.R. 1999. Identification of Enterococcus spp. with a biochemical key. Appl. Environ. Microbiol. 65:4425-4430p.

Marguet, E. R., Vallejo, M. y Olivera, N. L. 2008. Factores de virulencia de cepas de Enterococcus aisladas de quesos ovinos. Acta Bioquím. Clín. Latinoam. 42(4):543-548p.

Marín, M.E., Mera, J.R., Arduino, R.C., Correa, A.P., Coque, T.M., Stamboulian, D. and Murray, B.E. 1998. First report of vancomycin-resistan Enterococcus faecium isolated in Argentina. Clin. Infect. Dis. 26:235-236p.

Miranda, G., Corso, A., Melano, R., Arismendi, P., Rodríguez, M. and Garbervetsky, L. 2003. 1st isolation of vancomycin-resistant Enterococcus faecium with the vanB genotype in Argentina: presentation of 2 cases. Rev. Argent. Microbiol. $35(1): 41-4 p$.

Murray, B.E. 1990. The life and times of the Enterococcus. Clin. Microbiol. Rev. 3:46-65p.

Nilsson O. 2012. Vancomycin resistant enterococci in farm animals-occurrence and importance. Infect. Ecol. Epidemiology. 2:1-8p.

Ogier, J.C. and Serror, P. 2008. Safety assessment of dairy microorganisms: the Enterococcus genus. Int. J. Food Microbiol. 126: 291-301p. 
Rahimi, H., Gires, U. and Asmat, A. Antimicrobial activity of bacteria associated with various marine sources. 2013. Adv. Environ. Biology. 7(2):356-365p.

Rahimi, F., Talebi, M., Saifi, M. and Pourshafie M.R. 2007. Distribution of enterococcal species and detection of vancomycin resistance genes by multiplex PCR in Tehran Sewage. Iranian Biomedical Journal. 11(3):161-167p.

Rozdzinski, E., Marre, R., Susa, M., Wirth, R. and Muscholl-Silberhorn, A. 2001. Aggregation substance-mediated adherence of Enterococcus faecalis to immobilized extracellular matrix proteins. Microb. Pathog. 30:211-220p.

Semedo, T., Almeida Santos, M., Lopes, M.F., Figueiredo Marques, J.J., Barreto Crespo, M.T. and Tenreiro, R. 2003a. Virulence factors in food, clinical and reference Enterococci: a common trait in the genus? Syst. Appl. Microbiol., 26:13-22p.

Semedo, T., Almeida Santos, M., Martins, P., Silva Lopes, M.F., Figueiredeo Marques, J.J. Teneiro, R. et al. 2003b. Comparative study using type strains and clinical and food isolated to examine hemolytic activity and ocurrente of the cyl operon in enterococci. J. Clin. Microbiol. 41:2569-2576p.

Silva, N., Igrejas, G., Vaz, J., Araújo, C., Cardoso, L., Rodrigues, J., Torres, C. and Poeta, P. 2011. Virulence factors in enterococci from partridges (Alectoris rufa) representing a food safety problem. Foodborne Pathog. Dis. 8(7):831-833p.

Van den Bogaard, A.E. and Stobberingh, E.E. 2000. Epidemiology of resistance to antibiotics. Links between animal and human. Int. J. Antimicrob. Agents. 16:S327-S335p.

Werner G., Coque T.M., Hammerum A.M., Hope R, Hryniewicz, W., Johnson A, et al. 2008. Emergence and spread of vancomycin resistance among enterococci in Europe. Eurosurveillance. 13:1-11p.

\section{CORRESPONDENCIA}

Marisol Vallejo

Universidad Nacional de la Patagonia San Juan Bosco (UNPSJB). Roca 115, $1^{\circ}$ piso. TrelewChubut, Argentina.

soltrelew@yahoo.com.ar 